\title{
La homogeneidad social de la elite política. Los parlamentarios de los PANE (1980-2005)
}

\section{Xavier Coller}

Universidad Pablo de Olavide. Sevilla. Departamento de Ciencias Sociales xavier.coller@aya.yale.edu, xaviercoller@upo.es

\section{Andrés Santana}

Fundación Juan March

asantana@ceacs.march.es

\section{Resumen}

Los parlamentarios y las parlamentarias de los PANE, tomados en su conjunto, son sensiblemente diferentes a los de los partidos de ámbito estatal. Suelen incorporar menos mujeres en sus listas electorales en puestos de elección, menos inmigrantes internos, diputados de más edad, personas con credenciales educativas similares a las de los diputados de los partidos de ámbito estatal, menos educadores y trabajadores y más empresarios y directivos, aunque tienen cantidades similares de profesionales del derecho. Dentro de los PANE, hay partidos que muestran comportamientos distintos, lo cual aporta matices cuya diferencia conviene explicar.

Palabras clave: homogeneidad social, partidos políticos, elites políticas, perfil social.

Abstract: Social homophily in the political elite. Subnational MPs (1980-2005)

MPs from subnational parties (PANE) show a different social profile than those MPs from national parties elected for regional parliaments. They incorporate less women in parliaments, less internal migrants, older politicians, less workers and educators (but more owners and managers) and a similar proportion of lawyers. There are relevant differences among subnational parties the article analyzes thoroughly.

Key words: social homophily, political parties, political elites, social profile.

\section{Sumario}

Introducción Conclusiones y agenda de investigación:

Hombres, nativos, jóvenes y educados Homogeneidad social preguntas

Bibliografía 


\section{Introducción $^{1}$}

En los 25 años de funcionamiento de la España de las autonomías de los que se ocupa este trabajo, se han celebrado 107 elecciones autonómicas en las que 44 partidos políticos han obtenido 7.424 escaños que han ocupado 4.354 personas. Este grupo forma la elite de un poder político regional cuya relevancia crece a medida que se profundiza y se consolida el estado de las autonomías. Tal como se ha puesto de manifiesto en otros trabajos, llama la atención que no se haya realizado ningún estudio comparado de esta elite emergente. En este artículo, se sigue dicha tarea prestando atención especial a los parlamentarios de los partidos de ámbito no estatal (PANE) y estableciendo comparaciones relevantes con los partidos estatales que han obtenido representación parlamentaria en las cámaras autonómicas ${ }^{2}$.

El análisis que planteamos prescinde de cierto andamiaje teórico para centrarse en el estudio directo de los datos de parlamentarios de los PANE que, por vez primera, se ofrecen en una publicación española. Nuestro análisis está guiado por el objetivo de ofrecer respuestas a las preguntas siguientes: ¿existen diferencias sociales entre los diputados/as de estos partidos?, ¿hay elementos distintivos y comunes entre parlamentarios de los PANE y de los partidos de ámbito estatal?, ¿qué factores explican las diferencias y las similitudes entre ellos?, ¿las elites políticas son socialmente homogéneas o se caracterizan por su diversidad?, ¿existen diferencias entre partidos en lo que respecta al perfil social de sus representantes en los parlamentos? Para responder a estas cues-

1. Esta investigación ha sido posible gracias a la financiación del Ministerio de Educación y Cultura, proyecto BSO2003-02596, y también se benefició de los fondos con que la Fundación Endesa dota a la Cátedra Príncipe de Asturias, Georgetown University, que el primer autor ocupó entre 2005 y 2007. La mayor parte de los datos en los que se basa este trabajo están extraídos de las biografías oficiales de los diputados y las diputadas depositadas en las cámaras autonómicas. Los autores agradecen a varios funcionarios de diferentes parlamentos autonómicos, del Congreso de los Diputados y del Senado su ayuda para facilitar los datos biográficos en los que, en parte, se basa este trabajo. Se agradecen también las gestiones de José María Aznar, Germà Bel y Juan Manuel Eguiagaray para ayudar a obtener los datos necesarios para este estudio. Se agradece a Jeff Anderson, director del BMW Center for German and European Studies; a Jeff Alexander, director del departamento de Sociología de Yale Unviersity, y a los responsables del CEACS (Fundación Juan March), especialmente a su director José María Maravall, que nos facilitaran el uso de sus instalaciones para la elaboración de partes diferentes de este trabajo. El primer autor agradece también a Helder Ferreira Do Vale y a Chris Meissner su dedicación para construir las bases de datos que han hecho posible esta investigación. Albertino Robelló, Xavier Campmany, Neus Carreras, Sofía Marbán y Julio López también ayudaron en diferentes partes de la investigación. Se agradece también el interés y los comentarios de los asistentes a varias conferencias internacionales en las que se presentaron los resultados de esta investigación, y especialmente las contribuciones de los participantes en el taller del Institut de Ciències Politiques i Socials (Barcelona), dirigido por Montserrat Baras. Finalmente, se agradece las sugerencias de los evaluadores de la revista Papers. Para comentarios o críticas, se puede escribir al primer autor a xavier.coller@aya.yale.edu.

2. Para una información más completa sobre la totalidad de la elite política autonómica, véanse los trabajos de Coller y otros $(2007,2008)$ y Coller (2008). 
tiones básicas, nos centramos en el estudio del perfil sociográfico de los 4.354 diputados y diputadas y de su evolución, pero haciendo hincapié en los 795 (18\%) diputados elegidos en las listas de los PANE.

El análisis tiene que ir precedido de cinco advertencias al lector que evitarán errores de interpretación. En primer lugar, la formación del estado de las autonomías ha sido desigual, lo que ha dado origen a lo que se ha denominado "federalismo asimétrico" (Requejo, 1996), "federalismo imperfecto» (Moreno, 1997) o "semifederalismo" (Lijphart, 1999: 189). Aunque existen tendencias a la igualación de las competencias de todas las comunidades (unas tendencias que encuentran resistencias notables), hay un aspecto que, de momento, no ha sido afectado: la capacidad de los presidentes de los gobiernos autonómicos de Andalucía, Cataluña, Galicia y el País Vasco de disolver el Parlamento regional y convocar elecciones a su voluntad. El resto de presidentes de gobierno regional no dispone de esta prerrogativa. Ello hace que existan varios ciclos electorales, tal como se observa en la tabla 1 . La consecuencia para este trabajo es que la comparación de la evolución de los perfiles de los diputados corre el riesgo de ignorar, por ejemplo, que la tercera

Tabla 1. Elecciones y escaños en España

\begin{tabular}{|c|c|c|c|c|c|c|c|}
\hline & \multicolumn{7}{|c|}{ Legislaturas } \\
\hline & I & II & III & IV & V & VI & VII \\
\hline & Fecha Esc. & Fecha Esc. & Fecha Esc. & Fecha Esc. & Fecha Esc. & Fecha Esc. & Fecha Esc \\
\hline Andalucía & 1982109 & 1986109 & 1990109 & 1994109 & 1996109 & 2000109 & 2004109 \\
\hline Aragón & $1983 \quad 66$ & $1987 \quad 67$ & 199167 & 199567 & 199967 & $2003 \quad 67$ & - \\
\hline Asturias & $1983 \quad 45$ & $1987 \quad 45$ & $1991 \quad 45$ & $1995 \quad 45$ & $1999 \quad 45$ & $2003 \quad 45$ & - \\
\hline Canarias & $1983 \quad 60$ & 198760 & $1991 \quad 60$ & 199560 & $1999 \quad 60$ & $2003 \quad 60$ & -- \\
\hline Cantabria & $1983 \quad 35$ & $1987 \quad 39$ & $1991 \quad 39$ & 199539 & $1999 \quad 39$ & $2003 \quad 39$ & -- \\
\hline $\begin{array}{l}\text { Castilla } \\
\text { y León }\end{array}$ & 198384 & $1987 \quad 84$ & $1991 \quad 84$ & 199584 & 199983 & $2003 \quad 82$ & - \\
\hline Castilla- & & & & & & & \\
\hline La Mancha & $1983 \quad 44$ & $1987 \quad 47$ & $1991 \quad 47$ & $1995 \quad 47$ & $\begin{array}{ll}1999 & 47\end{array}$ & $2003 \quad 47$ & - \\
\hline Cataluña & 1980135 & 1984135 & 1988135 & 1992135 & 1995135 & 1999135 & 2003135 \\
\hline Comunidad & & & & & & & \\
\hline Valenciana & $1983 \quad 89$ & 198789 & 199189 & $1995 \quad 89$ & 199989 & 200389 & - \\
\hline Extremadura & $1983 \quad 65$ & $1987 \quad 65$ & $1991 \quad 65$ & 199565 & $1999 \quad 65$ & $2003 \quad 65$ & -- \\
\hline Galicia & $1981 \quad 71$ & 198571 & $1989 \quad 75$ & 199375 & $1997 \quad 75$ & $2001 \quad 75$ & 200575 \\
\hline Baleares & $1983 \quad 54$ & $1987 \quad 59$ & $1991 \quad 59$ & $1995 \quad 59$ & $1999 \quad 59$ & 200359 & - \\
\hline La Rioja & $1983 \quad 35$ & 198733 & 199133 & $1995 \quad 33$ & $1999 \quad 33$ & $2003 \quad 33$ & -- \\
\hline Madrid & $1983 \quad 94$ & 198796 & 1991101 & 1995103 & 1999102 & 2003111 & -- \\
\hline Murcia & $1983 \quad 43$ & $1987 \quad 45$ & $1991 \quad 45$ & $1995 \quad 45$ & $1999 \quad 45$ & $2003 \quad 45$ & - \\
\hline Navarra & $1983 \quad 50$ & $1987 \quad 50$ & $1991 \quad 50$ & $1995 \quad 50$ & $1999 \quad 50$ & 200350 & -- \\
\hline País Vasco & $1980 \quad 60$ & $1984 \quad 75$ & 198675 & 199075 & $1994 \quad 75$ & $1998 \quad 75$ & 200175 \\
\hline$\overline{\text { Congreso }}$ & 1979350 & 1982350 & 1986350 & 1989350 & 1993350 & 1996350 & 2000350 \\
\hline
\end{tabular}


legislatura en Andalucía comenzó cuatro años después que la del País Vasco, dos años después que la de Cataluña y un año antes que las de comunidades del régimen común. Este desfase temporal debe ser tenido en cuenta por el lector cuando se hable genéricamente de los cambios en el perfil social de los diputados y las diputadas.

En segundo lugar, ha sido inevitable hacer una selección de los PANE que serán analizados. De un total de PANE superior a 50, se ha escogido a siete partidos para un análisis más detallado: CiU (213 diputados), PNV-EA (123), CC (79), HB (71), ERC (50), PAR (49) y BNG (33). El criterio seguido ha sido doble. Por un lado, se ha seleccionado a estos partidos porque son los que han obtenido una representación parlamentaria mayor, lo que contribuye a hacer que los datos y los contrastes estadísticos presentados sean más fiables. Por otro lado, adoptando un criterio finalista, se ha primado a aquellos partidos que existen en la actualidad.

El resto de PANE se ha ubicado en la categoría «otros». Algunos PANE son como el Guadiana, aparecen y desaparecen en los parlamentos y, en muchas ocasiones, aún perdurando, obtienen una representación parlamentaria tan reducida durante el período estudiado que hemos optado por agruparlos en la categoría «otros». Es el caso, por ejemplo, de Tierra Comunera, Unión para el Progreso de Cantabria, Extremadura Unida, Partido Riojano, Unión Valenciana, Partido Andalucista, Partiu Asturianista o Euskadiko Eskerra. Su ubicación en la categoría «otros» sólo es producto del tamaño de su representación parlamentaria o de la duración de su existencia, no de su relevancia política, ya que algunos de estos partidos han gobernado o han tenido la llave de la gobernabilidad de algunas comunidades.

En tercer lugar, conviene hacer algunas especificaciones para no conducir a errores al lector. A efectos del análisis, hemos decidido fusionar los pocos parlamentarios de la Unión de Centro Democrático (UCD) con los del Centro Democrático y Social (CDS) en el grupo de partidos estatales, aun siendo conscientes de las diferencias, de su poca relevancia numérica (3\% de parlamentarios) y de la práctica extinción de ambas formaciones en la actualidad.

A lo largo del trabajo, se habla de Coalición Canaria (CC) como un partido que se presenta en todas las elecciones. Sin embargo, CC surge en 1993, tras la fusión de varios partidos (AGI, AHÍ, ATI, AM, ICAN, etc.) que se presentan en diferentes islas y que obtienen representación parlamentaria. A efectos del análisis, y dada la fusión de 1993 y la continuidad de la formación resultante, se ha optado por agrupar a todos estos partidos bajo la etiqueta $\mathrm{CC}$ en las elecciones a las que se presentaron antes de 1993.

De manera similar, dado que Unión del Pueblo Navarro (UPN) y Partido Popular (PP) se fusionan en 1991 y que UPN se convierte en la marca electoral del PP en Navarra, a efectos de contabilidad para este trabajo, se ha optado por agrupar a los diputados de UPN antes de la fusión bajo la etiqueta $\mathrm{PP}^{3}$.

3. Durante la revisión de este artículo, se acababa de producir la escisión de UPN y PP, evento que no se ha considerado en este trabajo. 
Se observará, también, que, para evitar problemas terminológicos, PP engloba a los parlamentarios de lo que fue Coalición Popular (y antes Alianza Popular o Coalición Democrática), formación que se convierte en el PP tras su refundación en 1989.

También se ha procedido de esta manera en el caso de Izquierda Unida (IU), etiqueta que se ha utilizado para los parlamentarios elegidos en las listas de los partidos que integraron esta coalición en 1986, muy especialmente los del Partido Comunista de España (PCE). También hemos utilizado el término Herri Batasuna (HB) o Euskal Herritarrok $(\mathrm{EH})$ para referirnos a los diputados electos en las formaciones herederas de $\mathrm{HB}$, al tener que cambiar ésta su nombre por su asociación con el grupo terrorista ETA.

En el caso del Partido Nacionalista Vasco (PNV) y Eusko Alkartasuna (EA), se ha optado por considerar ambos partidos conjuntamente. EA surge como una escisión del PNV en 1986, compite electoralmente con el PNV en varios comicios desde la segunda legislatura y se alía con este partido en otras elecciones y en el gobierno vasco. A pesar de algunas diferencias ideológicas, se ha considerado que, por la historia de relaciones entre las dos formaciones, se las podría agrupar a efectos del análisis, a pesar de que, en las elecciones autonómicas de marzo de 2009, compiten por separado.

No se ha adoptado el mismo criterio con los diputados del Bloque Nacional Popular Galego y Partido Socialista Galego (BNPG-PSG), formaciones que se integraron inicialmente en el Bloque Nacionalista Galego en 1982. A diferencia del caso PNV-EA, estos partidos gallegos abandonaron el BNG en 1983 y crearon la alianza electoral Unidade Galega (UG) con el partido Esquerda Galega (EG). Esta alianza compitió directamente con el BNG para la segunda legislatura de Galicia. Aunque, tras las elecciones que dieron lugar a la cuarta legislatura, los partidos de UG se integran en el BNG, se ha decidido no englobar en el BNG a los diputados elegidos bajo otras formaciones. El motivo es que, por un lado, estos partidos han sido competidores y, por otro, que, a pesar de la fusión tras las elecciones de 1993, no ha habido alianzas electorales entre BNG y UG o BNPG-PSG.

En cuarto lugar, conviene resaltar que se ha aplicado una concepción estricta del concepto de PANE. Así, se ha considerado al PSC como parte integrante del PSOE y, por lo tanto, como no PANE, y lo mismo para el resto de federaciones de partidos de ámbito estatal, como el caso de Eskerda Batua.

En quinto lugar, hay que advertir al lector sobre el hecho de que los datos de que se disponen son incompletos en el caso de los partidos vascos (PNVEA y HB-EH). Los esfuerzos que se han realizado para obtener las biografías de los parlamentarios de esta comunidad han sido vanos en la mayor parte de ocasiones. Para el resto de partidos, disponemos de datos bastante completos: $100 \%$ para el género, $86 \%$ para la edad, $86 \%$ para el lugar de nacimiento, $72 \%$ para el nivel educativo y $77 \%$ para la profesión. 


\section{Hombres, nativos, jóvenes y educados}

El perfil más frecuente entre los parlamentarios autonómicos de los PANE en el periodo considerado es el de un varón (83\%), nacido en la comunidad en la que es elegido (92\%), con cuarenta y cuatro años de edad media en el momento de entrar por vez primera en la cámara autonómica, con credenciales educativas universitarias (84\% de diplomados, licenciados o doctores) y de profesión educador $(22 \%$, incluyendo a profesores de universidad, de enseñanzas medias y primaria) o con un trabajo relacionado con el mundo del derecho (19\%, incluyendo abogados, jueces, fiscales, procuradores y similares). Este perfil es ligeramente diferente al del diputado que accede a su escaño en las listas de un partido de ámbito estatal, que tiende a ser mujer con más frecuencia $(22 \%)$, es más habitual que haya nacido fuera de la comunidad en la que consigue un escaño (17\%), acostumbra a ser un poco más joven cuando entra por vez primera en el Parlamento autonómico (cuarenta y dos años de media), tiene unas credenciales educativas similares a las del diputado de los PANE, y suele haber en sus filas proporcionalmente más educadores, más trabajadores, menos empresarios y directivos, y una cantidad similar de profesionales del derecho.

Esta fotografía fija corresponde a un periodo amplio, por lo que no se perciben los cambios notables que ha experimentado el perfil de la elite política autonómica. En este trabajo no nos referiremos a la evolución de este perfil, más allá de algunas pinceladas puntuales para explicar ciertas situaciones. Remitimos al lector interesado en esta evolución a otros trabajos (Coller y otros, 2007, 2008; Coller, 2008). De la misma manera, la foto fija no permite dilucidar si existen diferencias entre PANE o, incluso, si hay divergencias respecto de los partidos de ámbito estatal que concurren a las elecciones autonómicas. Para comprender estas diferencias, será conveniente analizar los datos resumidos para el periodo 1980-2005 que se presentan a partir de ahora.

Uno de los aspectos más interesantes en el análisis de la elite política autonómica es la diferencia que existe entre los PANE y los partidos de ámbito estatal en lo que respecta al género ${ }^{4}$. En la medida en que la presencia de mujeres en la vida política parlamentaria esté relacionada con el grado de apertura a la sociedad, parece que los PANE se han comportado de manera más cerrada que los otros partidos, ya que de las personas que han situado en los parlamentos autonómicos sólo el $17 \%$ son mujeres frente al $22 \%$ de los partidos estatales (PSOE, IU, UCD-CDS y PP). El hecho de que la diferencia sea estadísticamente significativa ${ }^{5}$ indica que ésta no es debida al azar y que, por tanto, los PANE han seguido con más frecuencia una política consistente en privilegiar a los varones frente a las mujeres a la hora de confeccionar las listas elec-

4. Sobre la presencia de la mujer en la política, véanse los trabajos de Mateo Díaz (2005), Norris y Lovenduski (1995), Uriarte y Ruiz (1999), Valiente, Ramiro y Morales (2003), Verge (2006) y Delgado y Jerez (2008).

5. $\mathrm{Chi}^{2}$ es 12,5207 y la probabilidad asociada es 0,001 . 
Tabla 2. Género de los parlamentarios/as según el partido político (1980-2005) (en \%)

\begin{tabular}{lrlllllllllrr}
\hline & \multicolumn{1}{c}{} & CiU & PNV-EA & CC & HB-EH & ERC & PAR & BNG & Otros & PANE & PANE & Total \\
\hline Mujeres & 14 & 28 & 14 & 17 & 14 & 12 & 30 & 12 & 17 & 22 & 21 \\
Hombres & 86 & 72 & 86 & 83 & 86 & 88 & 70 & 88 & 83 & 78 & 79 \\
\hline Total (N) & 213 & 123 & 79 & 71 & 50 & 49 & 33 & 177 & 795 & 3.559 & 4.354 \\
\hline
\end{tabular}

Fuente: elaboración propia a partir de fuentes oficiales.

$\mathrm{N}$ = número de casos válidos para los que se ha obtenido información.

torales y ubicar a unos y otros en puestos de elección segura. Puede ser, no obstante, que la oferta de candidatas sea inferior a la de candidatos para ser incorporadas a las listas, lo que convertiría la menor presencia de mujeres en los parlamentos en un efecto del sesgo muestral. Por una u otra razón, el resultado es que los varones muestran una mayor presencia en los PANE en comparación con los partidos estatales. En concreto, es 1,4 veces más probable que un varón entre en un parlamento regional en las listas de un PANE que lo haga una mujer en comparación con lo que sucede en los partidos estatales ${ }^{6}$.

Sin embargo, conviene matizar que, como se puede apreciar en los datos de la tabla 2, existen variaciones en el comportamiento de los PANE. PAR, $\mathrm{CC}$ y, sorprendentemente, ERC y CiU son los partidos que contribuyen proporcionalmente menos a que las mujeres accedan a puestos de representación durante el periodo. Por el contrario, quien incorpora proporcionalmente más mujeres a su cámara autonómica es el BNG, lo que en buena medida puede deberse a que su crecimiento electoral ocurre en las elecciones de 1993 (pasa de cinco diputados en la tercera legislatura a trece en la siguiente), cuando ya se había instalado en la cultura política española la idea avanzada por el PSOE de reservar ciertas cuotas para la presencia de mujeres en los órganos de dirección del partido y, por extensión, en las listas electorales.

En cambio, no puede ser ésta la explicación de la elevada presencia de mujeres en el grupo de diputados del PNV-EA, ya que, desde la primera legislatu$\mathrm{ra}$, estas formaciones incluyen una proporción de mujeres en el Parlamento vasco similar a la de los partidos de ámbito estatal ${ }^{7}$. A diferencia de otras formaciones, existe en el PNV-EA una cierta tradición de abrir las puertas de la política a las mujeres con más frecuencia (y desde antes) que en otros PANE, incluso vascos. Desde la primera legislatura, el PNV-EA sitúa en el Parlamento vasco proporcionalmente a más mujeres que otros partidos de ámbito estatal y

6. El cociente de probabilidades es 0,72 para mujeres en PANE y de 1,37 para hombres. Cuanto más se aproxime este estadístico a 1, más cerca de la independencia están las dos variables tenidas en cuenta (género y partido, en este caso).

7. La proporción de mujeres por legislatura en el PNV-EA es de $12 \%, 16 \%, 17 \%, 22 \%, 30 \%$, $30 \%, 50 \%$, bastante por encima de otros partidos de ideologías y ámbitos políticos diferentes (Coller y otros, 2008). 
otros competidores electorales como HB-EH, que es de los que también de forma continuada sitúa proporcionalmente menos mujeres en el Parlamento vasco (17\% en el periodo). Quizá habría que indagar en el mapa cognitivo de los líderes del PNV y de EA, pero especialmente de los selectorados, para averiguar por qué, a diferencia de otros partidos, son consistentemente más abiertos a la presencia de mujeres en sus filas parlamentarias.

PNV y CiU tienen similitudes en su ideario, trayectoria, ideología, visión de la política, que les ha llevado a ciertas alianzas para las elecciones europeas. Además, son equivalentes estructurales, en la medida en que han ocupado u ocupan posiciones de poder institucional similares en sus comunidades respectivas. Quizá por ello cabría esperar una contribución similar de diputadas a sus cámaras autonómicas. Los datos de la tabla 2 contradicen esta suposición. A diferencia del PNV-EA en el Parlamento vasco, CiU es uno de los partidos que aporta una proporción menor de mujeres al Parlamento catalán, por debajo de la media de los PANE y también a cierta distancia de los partidos de ámbito estatal ${ }^{8}$. Curiosamente, ésta es también la situación de ERC, con una proporción similar a la de CiU y también con cierta consistencia en privilegiar a los varones frente a las mujeres en los puestos de elección segura en las listas electorales ${ }^{9}$. En realidad, cuando se compara a CiU con su equivalente estructural vasco (PNV-EA), se observa que las mujeres tienen casi la mitad de probabilidades de entrar en la cámara autonómica de la mano de CiU que si lo hicieran en las listas del PNV-EA ${ }^{10}$. En trabajos anteriores, se detectó y se analizó esta situación concluyendo que en el nacionalismo catalán, a diferencia del vasco (y, por lo que se aprecia en la tabla 2, también del gallego), existe una cierta cultura política que actúa de freno a la participación de la mujer en puestos de representación política, al menos en el Parlamento catalán. Es cierto que la situación en 2005 es diferente a la de 1980 y que la proporción de diputadas de CiU y de ERC ha crecido. Para todo el periodo, la proporción es del 14\%. En la primera legislatura, era del $7 \%$ para CiU y del $0 \%$ para ERC (6\% para el PSC-PSOE, $12 \%$ para lo que fue el PSUC y $0 \%$ para el PP), mientras que en la sexta legislatura era del $14 \%$ y del $8 \%$ respectivamente (42\% para el PP, $33 \%$ para el PSC-PSOE e IC). Los resultados de esta comparación requieren una investigación que abunde en el credo ideológico y, sobre todo, en la percepción que los dirigentes de los partidos nacionalistas catalanes, y especialmente de sus selectorados, tienen acerca del papel de la mujer y del hombre en las esferas pública y privada.

8. Las diferencias entre CiU y PNV-EA respecto a la presencia de mujeres en los parlamentos autonómicos no puede ser explicada por el azar, ya que $\mathrm{Chi}^{2}$ es 8,5607 y su probabilidad asociada es 0,003 .

9. El test de $\mathrm{Chi}^{2}$ muestra que no hay diferencias estadísticamente significativas en lo que respecta a la presencia de mujeres en las listas de $\mathrm{CiU}$ y de ERC. $\mathrm{Chi}^{2}$ es 0,0101 y la probabilidad asociada es de 0,920 .

10. El cociente de probabilidades es 0,43 . 
Tabla 3. Nativos e inmigrantes internos según el partido político (1980-2005) (en \%)

\begin{tabular}{lrllllllllllrr}
\hline & \multicolumn{1}{c}{} & \multicolumn{1}{c}{ CiU } & PNV-EA & CC & HB-EH & ERC & PAR & BNG & Otros & PANE & PANE & Total \\
\hline Inmigrantes & 5 & 15 & 11 & 7 & 0 & 12 & 3 & 12 & 8 & 17 & 16 \\
Nativos & 95 & 85 & 89 & 93 & 100 & 88 & 97 & 88 & 92 & 83 & 84 \\
\hline Total (N) & 195 & 26 & 75 & 27 & 48 & 48 & 32 & 151 & 602 & 3.172 & 3.774 \\
\hline
\end{tabular}

Fuente: elaboración propia a partir de fuentes oficiales.

$\mathrm{N}$ = número de casos válidos para los que se ha obtenido información.

Parece esperable que, en elecciones de carácter regional o local, la proporción de nativos que configuran las listas de los partidos sea más elevada que la de aquellas personas que han nacido en otra comunidad diferente a la que residen (inmigrantes internos). Este pronóstico se cumple en todas las comunidades autónomas y en todos los partidos. Según los datos de la tabla 3, sólo un 16\% de los diputados electos en el periodo 1980-2005 han nacido en una comunidad que no es la que les elige. Sin embargo, hay diferencias estadísticamente significativas: la proporción de inmigrantes internos en los partidos de ámbito estatal dobla la de los PANE ${ }^{11}$. Los partidos de obediencia regional, en comparación con los partidos de ámbito estatal, suponen un freno a la participación institucional de inmigrantes internos en los parlamentos, de tal manera que la probabilidad de que una persona inmigrante vaya en las listas de un partido estatal para las elecciones autonómicas es más del doble $(2,36)$ que si fuera en un PANE ${ }^{12}$.

Este «efecto barrera» ocurre cuando la proporción de inmigrantes internos en la población crece durante el periodo: según los datos de los censos de 1981 y de 2001, la población que vive en una comunidad autónoma pero que ha nacido en otra pasa del $11 \%$ al $17 \%$. Como se ha visto en otros trabajos, el índice de desproporción social de los inmigrantes internos durante el periodo considerado muestra una clara infrarrepresentación de este sector de la población en la elite política (Coller, 2008), que es más clara en unos PANE en comparación con otros ${ }^{13}$. Se observa en la tabla 3 que el único partido cuyo comportamiento se aproxima a la media es el PNV-EA, aunque la escasez de datos sobre los parlamentarios vascos pone en tela de juicio esta excepcionalidad. Hecha esta salvedad, el Partido Aragonés Regionalista y Coalición Canaria son los que más se aproximan a la media, ya que apor-

11. $\mathrm{Chi}^{2}$ es 19,7947 y la probabilidad asociada es de 0,0001 .

12. El cociente de probabilidades es 2,36 para inmigrantes y no PANE y de 0,42 para inmigrantes y PANE.

13. El índice de desproporción social es una medida de la distancia entre la elite y la población. Responde a la fórmula siguiente: IDS = proporción del grupo en la elite $\div$ proporción del grupo en el electorado. El IDS pasa de 1,54 en 1981 a 0,59 en 2001, lo cual indica un claro retroceso de la presencia de inmigrantes internos en los parlamentos regionales en veinte años. 
tan proporcionalmente más inmigrantes internos a las cámaras autonómicas. Por el contrario, ERC, BNG y CiU son los que menos recurren a los inmigrantes internos.

Hay tres factores que pueden explicar esta situación. Primero, la estructura de la población. Allí donde residen menos inmigrantes es probable que la cantera de candidatos se vea limitada por ese flanco. Sin embargo, se da el caso de que en Cataluña, una de las comunidades (con Madrid) donde residen más inmigrantes internos, sus partidos recurren poco a este segmento de la población. Lo curioso es que este comportamiento es compartido con ligeras diferencias de intensidad por todos los partidos catalanes, ya sean nacionalistas o no ${ }^{14}$. Segundo, dado su componente nacionalista, algunos PANE pueden atraer a menos inmigrantes y, por tanto, incorporarlos menos a las listas. Tercero, las preferencias (explícitas o no) de los selectorados de algunos partidos, que pueden conducir a promover a los nativos frente a los inmigrantes para puestos políticos. Lo que parece claro es que los PANE, en comparación con los partidos estatales, han supuesto una barrera (y algunos PANE más que otros) para la incorporación de los inmigrantes internos a la política regional, especialmente si se comparan con los partidos de ámbito estatal.

Hay un elemento que no puede ser pasado por alto. En términos generales, el grupo de inmigrantes suele acoger a más mujeres (25\%) que el de nativos $(20 \%)$, siendo la diferencia lo suficientemente significativa estadísticamente para que no sea debida al azar ${ }^{15}$. En otras palabras, en comparación con una mujer nativa, las mujeres inmigrantes tienen más probabilidades (exactamente un 1,36 más) de estar en una lista para las elecciones autonómicas. Esta conclusión se cumple también cuando consideramos sólo los PANE, aunque con una intensidad mayor. En este caso, los PANE prefieren a varones nativos con más frecuencia que los partidos estatales, como se puede apreciar en la tabla 3. Sin embargo, cuando se trata de mujeres, los PANE sitúan en sus listas a mujeres inmigrantes con más frecuencia que a varones inmigrantes, de manera que es un 1,53 veces más probable que una mujer inmigrante entre en el Parlamento en la lista de un PANE en comparación con un varón inmigrante.

Tabla 4. Edad media al entrar en el Parlamento según el partido político (1980-2005)

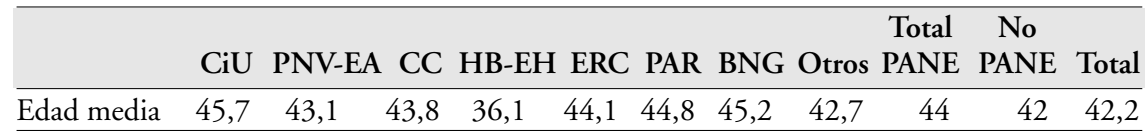

Fuente: elaboración propia a partir de fuentes oficiales.

14. Véase el trabajo de Coller y otros $(2007,2008)$. Para una explicación de esta «anomalía», se puede consultar el trabajo de Miley (2006).

15. $\mathrm{Chi}^{2}$ es 8,3830 y la probabilidad asociada es de 0,004 . 
La elite política española del periodo democrático suele ser más joven que sus homólogas de otros países europeos ${ }^{16}$, pero llaman la atención las ligeras diferencias que existen entre partidos en el caso de los PANE. Según los datos de la tabla 4, los representantes de partidos de ámbito estatal suelen ser dos años más jóvenes (de media) en el momento de entrar en las cámaras regionales que sus compañeros de los PANE. Esto indica que los PANE suelen situar en las listas electorales a candidatos ligeramente más viejos que los partidos estatales. Las diferencias entre partidos autonómicos son también notables. Los parlamentarios de más edad son los que aporta CiU (45,7 años de media en el momento de entrar por vez primera), y los más jóvenes, los de $\mathrm{HB}-\mathrm{EH}$ (36,1 años de media, aunque la carencia de datos para este partido convierte el resultado en poco fiable). La diferencia de medias entre PANE y partidos de ámbito estatal, aunque no es estadísticamente significativa, puede ser un indicador de algo curioso: quizá, los partidos de ámbito estatal ubican en sus listas a candidatos más jóvenes porque perciben a las cámaras regionales como estaciones de paso en la carrera política y un buen escenario donde adquirir experiencia legislativa y cintura política. Por contra, los PANE pueden percibir a la cámara autonómica como estación término y, consecuentemente, ubicar en sus listas electorales a candidatos que ya se han curtido en el ámbito local o en otras arenas políticas.

En términos generales, los hombres (43) suelen tener más edad que las mujeres (40) a la hora de acceder por vez primera a la cámara autonómica ${ }^{17}$. Pero donde las diferencias se hacen más grandes (y son también significativas) es en el grupo de los PANE en comparación con los partidos estatales. Los varones que acceden al Parlamento por vez primera en una lista de PANE son, de media, tres años más viejos (45) que las mujeres (42). Es más, mientras la edad de los varones decrece a medida que pasan las legislaturas, la edad media de las mujeres que entran por vez primera en el Parlamento aumenta.

Los parlamentarios autonómicos tienen niveles educativos similares a los de los diputados nacionales y europeos: un $85 \%$ son doctores, licenciados o diplomados universitarios, ubicándose en el segmento alto del sistema educativo (con una media de 3,56$)^{18}$. A efectos informativos, sólo un $4 \%$ se ha quedado en primaria, un $12 \%$ terminó los estudios de grado secundario y el $85 \%$ ha pasado por la universidad en una modalidad u otra, aunque, entre los universitarios, lo más frecuente es que el parlamentario autonómico tenga una licenciatura (59\%), especialmente más entre los diputados de los PANE que entre los de los partidos de ámbito estatal. No hay diferencias significativas entre los par-

16. Véanse los trabajos de Linz y otros (2000), Blondel (1973), Martínez y Méndez (2000), Norris y Lovenduski (1995).

17. La relación entre género y edad media es estadísticamente significativa $(t=5.9940$ y su probabilidad asociada es 0,0001).

18. Para una comparación del nivel educativo de los parlamentarios autonómicos con otros parlamentarios, véanse los trabajos de Norris (1999), Martínez y Méndez (2000), Genieys (2004), y los trabajos incluidos en el texto de Best y Cotta (2000). 
Tabla 5. Nivel educativo de los parlamentarios y parlamentarias según partido político (1980-2005)

\section{Total No}

CiU PNV-EA CC HB-EH ERC PAR BNG Otros PANE PANE Total

Educación

\begin{tabular}{lrrrrrrrrrrr} 
media & 3,56 & 3,82 & 3,68 & 2,89 & 3,9 & 3,32 & 3,81 & 3,79 & 3,64 & 3,54 & 3,56 \\
\hline Primaria (\%) & 0 & 3 & 0 & 26 & 0 & 0 & 0 & 2 & 2 & 4 & 4 \\
Secundaria (\%) & 14 & 3 & 6 & 11 & 6 & 32 & 14 & 9 & 11 & 12 & 12 \\
Diplomatura (\%) & 22 & 3 & 24 & 15 & 19 & 15 & 4 & 11 & 16 & 18 & 18 \\
Licenciatura (\%) & 59 & 88 & 62 & 44 & 53 & 43 & 67 & 65 & 62 & 58 & 59 \\
Doctorado (\%) & 6 & 2 & 6 & 4 & 21 & 11 & 14 & 14 & 9 & 8 & 8 \\
\hline Total (N) & 165 & 61 & 68 & 27 & 47 & 47 & 21 & 110 & 546 & 2.611 & 3.157 \\
\hline
\end{tabular}

Fuente: elaboración propia a partir de fuentes oficiales.

1. $\mathrm{N}$ = número de casos válidos para los que se ha obtenido información.

2. La educación media es el resultado de dar una puntuación a cada grado educativo, de manera que las personas que tenían hasta primaria han recibido 1; las de secundaria, 2; las diplomaturas, 3; las licenciaturas, 4, y los doctorados, 5. La media se ha computado en función de estas asignaciones numéricas. Téngase en cuenta, sin embargo, que, al tratarse de una variable originalmente codificada de manera ordinal, esta media debe ser tomada sólo de manera orientativa, ya que, en rigor, las escalas ordinales, al no informar sobre la distancia entre las categorías, no permiten realizar el cómputo de medias.

lamentarios de los PANE y los de los partidos estatales en términos porcentuales, aunque en la tabla 5 se aprecia que las credenciales educativas medias de los diputados de los PANE $(3,64)$ son ligeramente superiores a las de los parlamentarios de los partidos de ámbito estatal $(3,54)$.

Los parlamentarios con credenciales educativas más altas (de media) son los de ERC y BNG. El dato de los parlamentarios del PNV-EA no es muy fiable, dada la carencia de biografías. Los que tienen la media de credenciales más bajas son los del PAR y CiU, aunque conviene remarcar que todos se sitúan en el segmento educativo universitario. Destaca sobremanera la elevada proporción de doctores en ERC (21\%) y también, aunque menos, en el BNG (14\%). Sin duda, estas proporciones elevan la media de educación y muestra el tipo de cantera de políticos que nutren las listas electorales de estos grupos, muy probablemente, aunque no de manera exclusiva, personas dedicadas a la enseñanza en la universidad.

La media educativa es la misma para mujeres y hombres $(3,56)$, lo que indica que no hay un sesgo en cuanto al comportamiento de los partidos respecto a las credenciales educativas según el género. Sin embargo, las medias educativas varían un tanto cuando comparamos los PANE y los partidos estatales en función del género. En los partidos de ámbito estatal, no existen diferencias de nivel educativo medio entre mujeres y hombres $(3,5)$, mientras que, en los PANE, las parlamentarias tienen un nivel medio un poco más alto $(3,72)$ que sus compañeros de escaño $(3,64)$. 
Tabla 6. Perfil profesional de los diputados y las diputadas autonómicos españoles (1980-2005) (en \%)

\begin{tabular}{|c|c|c|c|c|c|c|c|c|c|c|c|}
\hline & $\mathrm{CiU}$ & PNV-EA & $\mathrm{CC}$ & HB-EH & ERC & PAR & BNG & Otros & $\begin{array}{c}\text { Total } \\
\text { PANE }\end{array}$ & $\begin{array}{c}\text { No } \\
\text { PANE }\end{array}$ & Total \\
\hline Educadores & 12,3 & 11,5 & 21,6 & 8,3 & 30,2 & 6,4 & 38,9 & 242 & 17,4 & 22,3 & 21,5 \\
\hline $\begin{array}{r}\text { Profes } \\
\text { dere }\end{array}$ & 16,8 & 39,3 & 16,2 & 19,4 & 9,3 & 17,0 & 16,7 & 19,4 & 19,2 & 18,1 & 18,3 \\
\hline & 32,4 & & & & 14,0 & 42,6 & 0,0 & & 22,3 & 11,6 & 13,5 \\
\hline Trabajadores & 4,5 & 6,6 & 5,4 & 38,9 & 2,3 & 2,1 & 11,1 & 8,1 & 7,6 & 11,9 & 11,1 \\
\hline $\operatorname{cotal}(\mathrm{N})$ & 165 & 61 & 68 & 27 & 47 & 47 & 21 & 110 & 546 & 2.611 & 3.157 \\
\hline
\end{tabular}

Fuente: elaboración propia a partir de fuentes oficiales.

Nota: $\mathrm{N}$ = número de casos válidos para los que se ha obtenido información.

Con estas credenciales educativas, no es de extrañar que las profesiones más habituales entre la elite política autonómica sean las liberales (médicos, ingenieros, arquitectos, abogados), entre las que destaca sobremanera la presencia de profesionales del derecho (18\%), tal como se puede apreciar en la tabla $6^{19}$. Aún así, la profesión más habitual en la elite política es la de educador $(21 \%)$, categoría que incluye a los profesores universitarios y los de otros grados educativos. Mientras los profesionales del derecho se distribuyen más o menos homogéneamente, lo cual provoca que no haya diferencias perceptibles entre PANE y partidos de ámbito estatal, en el grupo de educadores la situación es diferente: los PANE tienen una proporción significativamente más baja de educadores frente a los partidos de ámbito estatal. La diferencia es inversa cuando observamos la categoría de empresarios y directivos. En este caso, los PANE colocan en sus listas significativamente más empresarios y directivos que los partidos de ámbito estatal, exactamente el doble. Sin embargo, no hay muchas diferencias en lo que respecta a la categoría de trabajadores.

En conclusión, los educadores son los más frecuentes, pero más en los partidos de ámbito estatal que en los PANE; los profesionales del derecho son el segundo grupo profesional, pero está repartido, de manera que no hay diferencias significativas entre PANE y partidos estatales; el tercer grupo es el de empresarios y directivos, que se concentra más en los PANE que en los partidos de ámbito estatal, a diferencia de los trabajadores que, según los datos de la tabla 6 , se encuentran más frecuentemente en los partidos de ámbito estatal que en los PANE.

Estas diferencias no pueden esconder las variaciones que existen entre los propios PANE. El de los educadores es el grupo profesional más habitual, aunque son más frecuentes en los PANE autoproclamados nacionalistas de izquier-

19. Sobre la profesión de los políticos, véanse los trabajos de DiPalma y Cotta (1986) y, en general, los textos editados por Best y Cotta (2000). 
da (ERC y BNG), tal como se aprecia en la tabla 6. Dos motivos pueden ayudar a explicar esta situación. Puede que exista una afinidad electiva entre la misión pedagógica de todo docente y la misión «educativa» de los partidos nacionalistas, impelidos a llevar su mensaje de construcción de la nación a la población. Por otra parte, muchos profesores en España son también funcionarios públicos, lo que les convierte en claramente dispensables para dedicarse a la política y, sobre todo, evita el coste de la vuelta al trabajo docente tras la vida parlamentaria. En los estudios que se han hecho de las elites políticas, especialmente en España, los partidos de izquierda suelen concentrar a más educadores que los de derecha. Quizá por este motivo en los partidos de ámbito estatal (PSOE, IU, aunque también PP) es más frecuente la profesión de educador que entre los PANE en su conjunto ${ }^{20}$.

Los profesionales del derecho, como ocurre también entre los partidos de ámbito estatal, están más o menos homogéneamente repartidos entre los PANE, aunque llama la atención su escasa presencia en ERC y su abultada frecuencia en el PNV-EA. No obstante, la escasez de datos de los diputados vascos conduce a ser cautos sobre esta presencia mayor de profesionales del derecho. Lo habitual en toda elite política desde la Revolución Francesa es que, en buena medida, se nutra de profesionales de las leyes ${ }^{21}$. Existen razones de afinidad electiva y de dispensabilidad que lo explican, pero es un misterio, quizá explicado por la base social de la que se nutren las listas electorales, por qué hay tan pocos profesionales del derecho en ERC.

El de empresarios y directivos es un grupo profesional distintivo en los PANE, en comparación con los partidos de ámbito estatal, aunque tiende a encontrarse con más frecuencia en ciertos PANE, probablemente aquéllos que pueden ser clasificados como partidos más o menos conservadores, independientemente de su ideología más o menos nacionalista. Según los datos de la tabla 6, tal es el caso del PAR, que concentra proporcionalmente más empresarios y directivos en sus filas parlamentarias que cualquier otro partido de los considerados aquí. En este sentido, el comportamiento de PAR, CIU y CC se asemeja al del PP, que acumula un $18 \%$ de empresarios y directivos en sus filas parlamentarias, frente al 6\% del PSOE y al 3\% de IU. Si se tiene en cuenta la ideología de izquierdas que algunos grupos nacionalistas dicen defender, llama también la atención el 14\% de empresarios y directivos de ERC y la ausencia de los mismos en el BNG.

Los trabajadores son los más infrarrepresentados en la elite política autonómica durante el periodo, y su presencia ha ido descendiendo desde que comenzó la andadura autonómica. No obstante, hay un grupo en el que destaca su presencia (BNG) y otro en el que destaca su ausencia (ERC). Este contraste llama la atención si se tiene en cuenta que los trabajadores han sido, tra-

20. La diferencia es significativa entre IU, PSOE y PP. IU tiene un 35\% de educadores para todo el período. El PSOE congrega, para el periodo estudiado, un $28 \%$ de educadores en sus parlamentarios regionales, mientras que en el PP la proporción es de $16 \%$.

21. Véase Weber (1946: 94) y Eulau y Sprague (1964). 
dicionalmente, fuente de militancia y de parlamentarios con más frecuencia en los partidos de izquierda que en los conservadores. Por ejemplo, en el PSOE hay un $18 \%$ de parlamentarios que son trabajadores (17\% en IU), mientras que en el PP sólo se encuentra un 5\% de diputados en esta categoría. En este respecto, ERC se comporta como un partido próximo a los conservadores, con una proporción de trabajadores en sus filas parlamentarias incluso menor que la de sus homólogos conservadores, como es el caso de CiU.

Así pues, cada PANE parece tener algún grupo profesional que predomina $y$, aunque puede parecer arriesgado, quizá convenga prestar atención a la composición interna (especialmente en lo que se refiere a las profesiones) para entender mejor y explicar el comportamiento de los partidos en las cámaras autonómicas y en las competiciones electorales. En CiU y el PAR predominan los empresarios y los directivos, mientras que en el PNV-EA (con la prevención conocida) predominan los profesionales del derecho. En CC también predominan los profesionales del derecho, aunque con una presencia importante de educadores. ERC parece ser un partido fundamentalmente de educadores, al igual que BNG.

\section{Homogeneidad social}

Es un lugar común en la sociología entender que la «homofilia» está en la base de diferentes procesos (comunicativos, políticos, de intercambio económico, etc.) en los que los individuos tienden a asociarse con aquellas personas que perciben como similares en términos de rasgos personales (género, edad, experiencias vitales), opiniones, actitudes, valores o posición social ${ }^{22}$. Un grupo altamente homofílico u homogéneo está también más cerrado a influencias exteriores y, por tanto, es más proclive a la autoafirmación, al consenso, a la endogamia intelectual y social y, por tanto, a desarrollar una visión de la realidad limitada y generalmente inmune a la crítica. Un grupo heterogéneo puede que incorpore experiencias, valores, opiniones, rasgos sociales diversos que generen puntos de vista contrapuestos, conflicto, pero también intercambio, renovación, novedad, pacto. En este apartado, intentamos hacer una aproximación al grado de homofilia u homogeneidad social que tienen los grupos parlamentarios de diferentes partidos, subrayando los PANE ${ }^{23}$.

El punto de partida es que un partido cuya representación parlamentaria sea diversa, probablemente afronta a las tareas legislativas con un acervo de cono-

22. Se utiliza el término "homofilia» como una traducción directa del término inglés homophily, con connotaciones diferentes a las del término homophile. La "homofilia» (del griego, afinidad, amor, afición, simpatía hacia las personas que consideramos semejantes o iguales) es una característica de las redes sociales y se suele utilizar como variable explicativa de otros fenómenos sociales (McPherson y otros, 2001, Ibarra, 1995).

23. En futuros trabajos se trasladará esta aproximación a los parlamentos y se intentará averiguar sus correlatos. De momento, nos conformamos con comprobar la utilidad del índice de homogeneidad que presentamos aquí. 
cimientos, puntos de vista, experiencias, contactos, actitudes diferentes a los de uno cuya representación sea homogénea. De la misma manera, la heterogeneidad social en una representación parlamentaria puede indicar una mayor preocupación por parte de los selectorados por reflejar las líneas de división (cleavages) de cualquier sociedad, ya sean éstas de naturaleza lingüística, de género, étnica, religiosa, de edad, ideológica o de cualquier otro tipo.

Aunque puede haber varias maneras de construir la noción de homogeneidad social para su contraste empírico, hemos optado por elaborar un índice que nos permite sintetizar la información de las variables que se han analizado: género, lugar de nacimiento, edad, estudios y profesión. Se trata de un índice ad hoc, sintético e intuitivo que nos permite analizar y clasificar el comportamiento de los partidos en el periodo 1980-2005, de manera que podemos identificar a las elites políticas con mayor o menor tendencia a la homofilia, es decir, más similares en sus rasgos sociales básicos. Cuando el índice se aproxime a 100, el grupo será más homogéneo y cuanto más se aproxime a 0 , habrá más diversidad en sus filas parlamentarias.

Todos los partidos incorporan sistemáticamente un porcentaje mayor de hombres que de mujeres, de nativos que de inmigrantes internos, y de licenciados que de cualquier otro nivel educativo. Por lo tanto, la representación parlamentaria de un partido será tanto más homogénea en términos de la composición de género, de origen y de estudios de sus diputados cuanto mayor sea el porcentaje de varones, nativos y licenciados, respectivamente. Estos tres porcentajes se han utilizado directamente para computar el índice de homogeneidad de los diputados de los partidos.

En cuanto a la edad, dado el carácter continuo de la variable, el cálculo de un porcentaje similar al de las otras variables supone considerar intervalos en lugar de una edad concreta. El procedimiento utilizado aquí ha sido el de calcular el porcentaje de diputados comprendido en un «núcleo de edad» que hemos ubicado en torno a la edad media de cada partido según se observa en la tabla 4. Este intervalo o núcleo de edad se ha construido tomando como límite inferior la media de la edad del partido menos la desviación estándar (9,6 años) del conjunto de partidos y como límite superior la media más una desviación estándar ${ }^{24}$.

24. El intervalo podría haberse construido con otras amplitudes (sumando y restando dos desviaciones estándar en vez de una, para hacerlo más ancho, por ejemplo). Lo fundamental no es tanto qué amplitud se escoge, ya que se tratará siempre de una opción particular, sino de que la misma opción sea aplicada a todos los partidos. La opción escogida aquí permite observar variaciones de homogeneidad en edad entre un 56,5\% (PNV) y un $89,3 \%$ (BNG). Se ha utilizado la desviación estándar del conjunto de partidos, y no de cada partido, por dos motivos: en primer lugar, uno técnico, a saber, que, de optar por la de cada partido, se estaría midiendo la asimetría, no la dispersión, ya que ésta sería de una desviación estándar para todos; en segundo lugar, uno conceptual, a saber, que, si un partido tiene mayor variabilidad en la edad de sus diputados, no se quiere perder esta información debido a la utilización de desviaciones estándar para cada partido, las cuales serían tanto mayores cuanto mayor fuera la variabilidad de edad. 
Finalmente, la medida de homogeneidad en cuanto a la profesión se ha calculado teniendo en cuenta (1) la proporción de diputados concentrados en los cuatro grupos profesionales discutidos más arriba (educadores, profesionales del derecho, empresarios y directivos, y obreros), que son los más habituales en la elite parlamentaria autonómica; y (2) la homogeneidad o la concentración de sus señorías dentro de estos cuatro grupos. La primera se obtiene directamente de la tabla 6 . Para calcular la segunda, se ha procedido a realizar una serie de operaciones que nos ha conducido a un indicador de homogeneidad profesional dentro de cada partido (la medida utilizada es «1-media geométrica» de las cuatro profesiones) ${ }^{25}$.

Con los datos de homogeneidad en las cinco dimensiones comentadas, se puede proporcionar un índice sintético de homofilia. El índice más simple e intuitivo consiste en la media aritmética de los valores de cada uno de los cinco componentes indicada en la tabla 7. También se ha calculado una media geométrica (que da lugar a valores menores de homogeneidad si ésta se concentra en dimensiones específicas, a costa de mayor diversidad en otras dimensiones) y una media ponderada. Dicha media ponderada es inversamente proporcional a la variabilidad de cada dimensión, es decir, pondera más las dimensiones que varían menos y pondera menos las dimensiones que varían más ${ }^{26}$. Sin embargo, los resultados son prácticamente idénticos, independientemente de cual de las tres opciones se escoge.

En cualquiera de los tres casos, el índice puede variar entre 0 y 100 . Un valor próximo a 100 quiere decir que los parlamentarios del grupo en cuestión son muy similares en términos de su edad, género, lugar de nacimiento, estudios y profesión. Por el contrario, si el índice se aproxima a 0 , indica que hay una dispersión máxima y que los parlamentarios son prácticamente diferentes. Por cuestiones obvias, 0 y 100 son valores extremos e imposibles. Lo normal es encontrarse con ciertas similitudes y con ciertas diferencias, pero lo

25. En primer lugar, se han recalculado los coeficientes de la tabla 6 sobre el total de estas cuatro categorías profesionales, para que estas cuatro categorías sumen un $100 \%$. Por ejemplo, para el caso de ERC, se ha hecho el sumatorio de 0,30/0,56+0,09/0,56 + 0,14/0,56 $+0,02 / 0,56$, y se obtenido el valor «1» en aproximación por redondeo. En todos los partidos se obtiene el mismo resultado al realizar el sumatorio indicado. Esta operación garantiza, por definición, que la media aritmética de las cuatro categorías sea igual $(0,25)$ para todos los partidos. A continuación, se ha tomado la media geométrica de los porcentajes de cada uno de los grupos profesionales para cada partido. La media geométrica se calcula multiplicando los porcentajes de las cuatro profesiones y tomando la raíz cuarta del producto. En el caso de ERC, la media geométrica es 0,18. El máximo de esta media geométrica es 0,25 , cuando el porcentaje de diputados se reparte por igual entre las cuatro categorías profesionales. Cuanto menos equitativamente se repartan los diputados entre estas cuatro profesiones, menor será la media geométrica. Por tanto, dada una media aritmética constante, la media geométrica puede interpretarse como una medida de distribución equitativa entre grupos. Por este motivo se ha tomado «1-media geométrica» como medida de homogeneidad dentro de este grupo de cuatro profesiones.

26. Dicha variabilidad se ha calculado sumando los valores absolutos de las diferencias entre la media de cada partido y la media total. 
Tabla 7. Índice de homogeneidad social de la elite parlamentaria autonómica (19802005)

\begin{tabular}{lcccccccc}
\hline & $\begin{array}{c}\% \\
\text { hombres }\end{array}$ & $\begin{array}{c}\% \\
\text { nativos }\end{array}$ & $\begin{array}{c}\% \\
\text { licenciados }\end{array}$ & $\begin{array}{c}\% \text { edad } \\
\text { núcleo }\end{array}$ & $\begin{array}{c}\% \\
\text { profesión }\end{array}$ & $\begin{array}{c}\text { Media } \\
\text { aritmética }\end{array}$ & $\begin{array}{c}\text { Media } \\
\text { geométrica }\end{array}$ & $\begin{array}{c}\text { Media } \\
\text { ponderada }\end{array}$ \\
\hline PSOE & 74,7 & 82,8 & 51,7 & 76,6 & 50,2 & 67,2 & 65,8 & 67,6 \\
PP & 79,3 & 83,4 & 66,9 & 66,8 & 49,6 & 69,2 & 68,1 & 68,8 \\
IU & 77,9 & 79,5 & 50,6 & 76,4 & 53,1 & 67,5 & 66,2 & 68,0 \\
CDS-UCD & 96,4 & 78,4 & 67,0 & 75,8 & 52,9 & 74,1 & 72,7 & 73,6 \\
CiU & 85,5 & 95,4 & 58,2 & 63,7 & 52,8 & 71,1 & 69,3 & 71,6 \\
PNV-EA & 72,4 & 84,6 & 88,5 & 56,5 & 52,1 & 70,8 & 69,3 & 69,4 \\
CC & 86,1 & 89,3 & 61,8 & 69,3 & 54,1 & 72,1 & 70,8 & 72,3 \\
HB & 83,1 & 92,6 & 44,4 & 73,1 & 57,9 & 70,2 & 67,9 & 71,6 \\
ERC & 86,0 & 100,0 & 53,2 & 59,2 & 46,0 & 68,9 & 65,9 & 69,5 \\
PAR & 87,8 & 87,5 & 42,6 & 66,7 & 58,1 & 68,5 & 66,1 & 70,0 \\
BNG & 69,7 & 96,9 & 66,7 & 89,3 & 66,7 & 77,8 & 76,8 & 78,4 \\
Otros & 88,1 & 87,4 & 64,6 & 76,2 & 53,3 & 73,9 & 72,6 & 73,8 \\
PANE & 85,3 & 89,6 & 62,2 & 68,9 & 51,2 & 71,4 & 70,0 & 71,5 \\
No PANE & 77,0 & 82,8 & 58,3 & 71,4 & 48,6 & 67,6 & 66,4 & 67,6 \\
\hline Total & 78,8 & 84,1 & 59,1 & 71,8 & 48,8 & 68,5 & 67,2 & 68,5 \\
\hline
\end{tabular}

Fuente: elaboración propia a partir de fuentes oficiales.

intrigante es ver de qué manera la estructura social de la elite política tiende hacia la homofilia o si, por el contrario, tiende a la diversidad, y en qué medida los partidos políticos presentan comportamientos diferenciados.

Como se observa en la tabla 7 , el conjunto de partidos presenta índices de homofilia elevados, cercanos al 70. Esto indica que sus señorías son bastante parecidas en cuanto a las cinco características sociales básicas. Obviamente, este índice esconde diferencias temporales que hemos aparcado para tratarlas en otros trabajos, ya que el objetivo aquí es el de comprobar la utilidad de este índice para describir, clasificar y detectar diferencias en la población observada. Aún así, se puede ver que el comportamiento de los partidos de ámbito estatal es sensiblemente diferente al de los PANE. Éstos suelen ser más homogéneos socialmente que aquéllos, lo que sugiere que, bajo condiciones similares, los partidos estatales han introducido proporcionalmente una mayor variedad en el perfil social de sus parlamentarios: hay más mujeres, más inmigrantes internos, más diversidad profesional y también educativa, aunque menos personas fuera del núcleo de edad. No obstante, no todos los partidos estatales se comportan de manera similar. Los que podríamos llamar "de izquierda» (IU y PSOE) tienen índices de homogeneidad más bajos que los partidos llamados «de centro", "de centro derecha» o "de centro reformista» (UCD-CDS y PP). Esto indica que los parlamentarios de izquierdas suelen ser más diversos socialmente que los conservadores, quienes suelen ser más parecidos y homogéneos. 
Entre los PANE hay también comportamientos diferenciados. Los partidos cuyas representaciones parlamentarias son socialmente más homogéneas son $\mathrm{BNG}, \mathrm{CC}$ y $\mathrm{CiU}$, mientras que los que presentan más diversidad son ERC y PAR. Nótese que los partidos autoproclamados nacionalistas de izquierdas tienen un comportamiento más alejado de los partidos estatales de izquierda y más próximo al de los partidos estatales más conservadores, como se puede observar en la tabla 7.

El caso del BNG es extraordinario, porque presenta el índice de homogeneidad más elevado, quizá debido a dos factores. Primero, exceptuando a ERC, es el partido que tiene una proporción de nativos más elevada, lo que indica que este partido (junto a CiU y ERC) es de los más refractarios a la incorporación de inmigrantes internos por el motivo que sea. Segundo, se trata de un grupo cuyos parlamentarios se ubican mayoritariamente en el intervalo de edad definido como núcleo, lo que sugiere poca dispersión y variación. A pesar de que una proporción muy elevada (la más grande, 39\%) de parlamentarios del BNG son educadores - lo que indica también una gran concentración profesional y poca heterogeneidad-, el índice de homogeneidad profesional es más bajo de lo esperado.

En resumen, el índice de homogeneidad social de las representaciones parlamentarias permite clasificar a los partidos en un continuo para establecer comparaciones. Los PANE suelen ser más homogéneos que los partidos de ámbito estatal, lo que puede tener también ciertas consecuencias en términos de actividad legislativa, conexión con el electorado (con su secuela de apatía o implicación política) y percepción del Parlamento como una institución más o menos próxima a la sociedad.

\section{Conclusiones y agenda de investigación: preguntas}

Las delegaciones parlamentarias de los PANE, tomadas en su conjunto, son sensiblemente diferentes de las de los partidos de ámbito estatal. Suelen incorporar menos mujeres, menos inmigrantes internos, diputados mayores, personas con credenciales educativas similares a las de los diputados de los partidos de ámbito estatal, menos maestros y trabajadores y más empresarios y directivos, con niveles similares de profesionales del derecho. En términos generales, ésta es la fotografía de las diferencias entre diputados de los PANE y de los partidos estatales.

Sin embargo, cuando se observan los PANE individualmente, se aprecia que, en la fotografía, hay partidos que aportan una mayor intensidad de color que otros, y se descubren matices interesantes. Por ejemplo, CiU y ERC (junto a $\mathrm{CC}$ y $\mathrm{PAR}$ ) son los que contribuyen con menos mujeres a la elite política autonómica, mientras que PNV-EA y BNG son los que más diputadas colocan en los parlamentos. ¿Por qué existe este comportamiento diferenciado? ERC, CiU y BNG tienen los grupos parlamentarios que presentan proporciones menores de inmigrantes internos en sus filas, mientras que PAR y CC son los que más. ¿A qué obedecen estas diferencias? CiU y BNG tienen par- 
lamentarios con una edad media bastante superior a la del resto en el momento de entrar por vez primera en la cámara autonómica. ¿Hay algún motivo que pueda explicar esta situación?

El nivel educativo de los parlamentarios autonómicos es similar al de otras elites políticas, por lo que no existen diferencias notables entre los PANE. Sin embargo, en términos profesionales, el comportamiento es diferente. CiU y PAR tienen la presencia más frecuente de empresarios y directivos, mientras que ERC y BNG se nutren con más frecuencia que otros grupos del sector de la enseñanza. En el PNV-EA abundan los profesionales del derecho con más intensidad que en otros grupos, mientras que en CC tienen una presencia importante tanto los educadores como los profesionales del derecho. ¿Por qué los partidos políticos se nutren de profesionales diferenciados (excepto en el caso de los profesionales del derecho, que se ubican por igual en casi todos los partidos) mostrando ciertas preferencias por unas profesiones frente a otras?

Las microdecisiones que conducen a la confección de una lista electoral determinada que, al final, contribuirá a tener un parlamento predominantemente masculino, nativo, joven y educado, son de relevancia fundamental para entender no sólo la estructura social de la elite política o del Parlamento en cuestión, sino también para entender las «barreras» estructurales (aunque también ideológicas) que impiden a personas de ciertos grupos acceder a la representación política y los "catalizadores» que terminan privilegiando a personas con ciertas características frente a otras.

Estas reflexiones conducen a algunas preguntas que convendrá responder, pero en otros trabajos: ¿qué consecuencias tiene para la acción legislativa que los grupos parlamentarios (es decir, la cámara regional) sean similares o diversos en términos sociales?, ¿̨podemos presumir que habrá más consenso generado por una mayor homogeneidad?, ¿podemos intuir que cuanta mayor sea la diversidad de perfiles sociales, mayores probabilidades hay de tener parlamentos conflictivos? En otro orden de cosas, ¿es posible atribuir la desafección política de la ciudadanía a la homogeneidad relativa de los representantes políticos y su distancia con la sociedad? Es decir, ¿̇tener parlamentos que reflejen las líneas de división que estructuran una sociedad tiene ventajas para la implicación política de la ciudadanía? Teniendo en cuenta que muchas de las diferencias que se observan en el perfil social de los diputados no son debidas al azar, ¿es posible capturar los criterios latentes que se utilizan para confeccionar las listas electorales?, ¿cuáles son los mapas cognitivos de los selectorados de los partidos políticos, las carencias de la oferta (sobre todo en términos de género y lugar de nacimiento), y otros factores estructurales (por ejemplo, leyes que obligan a cuotas) que explican la composición final de las listas electorales que, a fin de cuentas, son el billete de entrada a cualquier Parlamento en sociedades democráticas? ${ }^{27}$. 


\section{Bibliografía}

BEST, Heinrich; COTTA, Maurizio (eds.) (2000). Parliamentary Representatives in Europe 1848-2000. Legislative recruitments and Careers in Eleven European Countries. Oxford: Oxford University Press.

CAlvet, Jordi (1999). "Les elits polítiques a Catalunya. Un estudi sociològic i polític dels diputats de la cinquena legislatura». Autonomies. Revista Catalana de Derecho Público, n. ${ }^{\circ}$ 24, p. 235-64.

Coller, Xavier (1999). "Circulación y conflicto en la elite política: el caso valenciano». Revista Valenciana de Estudios Autonómicos, n. ${ }^{\circ} 29$, p. 193-221.

- (2002). "Continuidad y conflicto parlamentario en las comunidades autónomas españolas». En: Joan Subirats y Raquel GALLEGO (eds.). Veinte años de autonomías en España: Leyes, politicas públicas, instituciones y opinión pública, Madrid: Centro de Investigaciones Sociológicas, p. 69-97.

- (2004). "Los políticos valencianos en el contexto español». Revista Valenciana de Estudios Autonómicos, no 41/42, p. 96-145.

- (2006). «Regional Political Elites in Newly Federalized Countries. The Case of Spain (1980-2005)». Penn-Temple European Studies Colloquium. Department of Political Science, University of Pennsylvania, <http://www.ptesc.ssc.upenn.edu/ Paper_pdf/Xavier_Upenn_social_background.pdf $>$.

- (2008). «El sesgo social de las elites políticas. El caso de la España de las autonomías (1980-2005)». Revista de Estudios Políticos, n. ${ }^{\circ}$ 141, p. 133-59.

Coller, Xavier; Ferreira, Helder; Meissner, Chris (2007). «Political Elites in Federalized Countries: The Case of Spain (1980-2005)». Center for European Studies Working Papers Series, n. ${ }^{\circ}$ 146, Harvard University, <http://www.ces. fas.harvard.edu/publications/ces_papers.html>.

— (2008). «Les elites politiques régionales espagnoles (1980-2005)». En: William Genieys, Mohammad-Saïd Darviche y Guy Hermet (eds.). Penser la dynamique des regimes politiques. Sur les pas de Juan Linz. París: L'Harmattan, p. 113-140.

DELGADO, Irene; JEREZ, Miguel (2008). «Mujer y política en España: un análisis comparado de la presencia femenina en las asambleas legislativas (1977-2008)». Revista Española de Ciencia Política, n. ${ }^{\circ}$ 19, p. 41-78.

Di Palma, Giuseppe; CotTa, Maurizio (1986). «Cadres, Peons, and Entrepreneurs: Professional Identities in a Divided Parliament». En: Ezra N. Suleiman, Parliaments and Parliamentarians in Democratic Politics. Nueva York: Holmes \& Meier, p. 41-78.

Eulau, Heinz; Sprague, John D. (1964). Lawyers in Politics: A Study in Professional Convergence. Indianapolis: Bobbs-Merrill Co.

GENIEYS, William (2004). Las élites españolas ante el cambio de régimen político: lógica de estado y dinámicas centro-periferias en el siglo XX. Madrid: CIS.

IBARRA, Herminia (1995). «Race, Opportunity, and Diversity of Social Circles in Managerial Networks». Academy of Management Journal, n.o 38, p. 673-703.

Instituto Nacional de Estadística (INE) (1985). Censo de población de 1981. Madrid: INE.

Instituto Nacional de EstadístiCa (INE). Censo de población de 2001, <www.ine.es>. Lijphart, Arend (1999). Patterns of Democracy. Government Forms and Performance in Thirty-Six Countries. New Haven: Yale University Press.

LINZ, Juan J.; JEREZ, Miguel; CORZO, Susana (2003). «Ministers and regimes in Spain: From the First to the Second Restoration, 1874-2002». En: Pedro TAVARES DE Almeida, António Costa PInTo y Nancy BermeO, Who Governs Southern Europe? 
Regime Change and Ministerial Recruitment, 1850-2000. Londres: Frank Cass Publishers, p. 41-116.

LinZ, Juan J.; JereZ, Miguel; GANGAS, Pilar (2000). «Spanish Diputados: From the 1876 Restoration to Consolidated Democracy». En: Heinrich BEST y Maurizio COTTA (eds.). Parliamentary Representatives in Europe 1848-2000. Legislative recruitments and Careers in Eleven European Countries. Oxford: Oxford University Press, p. 371-462.

LOPEZ NIETO, Lourdes (1997). «Reclutamiento y profesionalización política: reflexiones sobre los parlamentarios españoles». Perfiles Latinoamericanos, n. ${ }^{\circ} 11$, p. 139-56.

Mateo DíAz, Mercedes (2005). Representing Women? Female legislators in West European Parliaments. Essex: ECPR Press.

McPherson, Miller; SMith-Lovin, Lynn; Cook, James M. (2001). «Birds of a Feather: Homophily in Social Networks». Annual Review of Sociology, vol. 27, p. 415-44.

Miley, Jeff (2006). Nacionalismo y política lingüistica. El caso de Cataluña. Madrid: Centro de Estudios Políticos y Constitucionales.

Moreno, Luis (1997). La federalización de España. Madrid: Siglo XXI.

NORRIS, Pippa; LOVEnduSKI, Joni (1995). Political Recruitment. Gender, Race and Class in the British Parliament. Nueva York: Cambridge University Press.

NorRIS, Pippa (1999). «Recruitment into the European Parliament». En: Richard S. KATZ y Bernhard Wessels (eds.). The European Parliament, the National Parliaments, and European Integration. Oxford: Oxford University Press, p. 86-102.

REQUEJO, Ferran (1996). «Diferencias nacionales y federalismo asimétrico». Claves de Razón Práctica, n. ${ }^{\circ}$ 59, p. 24-37.

URIARTE, Edurne; RUIZ, Cristina (1999). «Mujeres y hombres en las elites políticas españolas: ¿¿diferencias o similitudes?». Revista Española de Investigaciones Sociológicas, n. ${ }^{\circ} 88$, p. $207-32$.

Valiente, Celia; Ramiro, Luis; Morales, Laura (2003). «Mujeres en el Parlamento: un análisis de las desigualdades de genero en el Congreso de los Diputados». Revista de Estudios Políticos, n. ${ }^{\circ}$ 121, p. 179-208.

VERGE, Tania (2006). «Mujer y partidos políticos en España: las estrategias de los partidos y su impacto institucional, 1978-2004». Revista Española de Investigaciones Sociológicas, n. ${ }^{\circ} 115$, p. 165-96.

Weber, Max (1946). «Politics as Vocation». En: Hans H. GerTH y Charles Wright Mills (eds. y trad.). From Max Weber: Essays in Sociology. Nueva York: Oxford University Press, p. 77-128. 